\title{
Alliance for Water Stewardship: A Network of Sustainable Water Stewards in Italy
}

\author{
Matteo Magagni ${ }^{1}$, Chiara Rizzi ${ }^{1}$, Maria Giuseppina Iannacci ${ }^{1}$, Eugenio Capponi $^{2}$, Beatrice Bizzaro ${ }^{2}$ \\ ${ }^{1}$ Philip Morris Manufacturing \& Technology Bologna S. p. A., Crespellano, Bologna, Italy \\ ${ }^{2}$ HPC Italia S. r. 1., Milan, Italy
}

Email address:

eugenio.capponi@hpc.ag (E. Capponi), beatrice.bizzaro@hpc.ag (B. Bizzaro)

\section{To cite this article:}

Matteo Magagni, Chiara Rizzi, Maria Giuseppina Iannacci, Eugenio Capponi, Beatrice Bizzaro. Alliance for Water Stewardship: A Network of Sustainable Water Stewards in Italy. Hydrology. Vol. 8, No. 4, 2020, pp. 98-103. doi: 10.11648/j.hyd.20200804.16

Received: February 20, 2020; Accepted: March 4, 2020; Published: December 8, 2020

\begin{abstract}
Overexploitation and competitiveness over shared freshwater resources are some of the growing problems that affect many regions of Italy, especially Emilia Romagna, a region characterized by high water-related risks linked to water scarcity, droughts and baseline water stress. Private and public sectors are driven to find alternative or best-practice strategies to conserve and optimize the water resource and guarantee a future use to all. The Alliance for Water Stewardship (AWS) is the first international standard that assesses water-use across a 5-step certification procedure, with the aim of promoting good water stewardship by assessing and consequently mitigating the physical, reputational and regulatory water-related risks associated to a certain site and its territory. The AWS is proving to be an innovative certification adopted by a wide range of multinational companies in order to reduce their water footprint. The Italian affiliate of Philip Morris Manufacturing and Tecnology Bologna (MTB) of Crespellano has, with support of the INOGEN International Alliance and environmental consultants Denkstatt and HPC, implemented the Alliance for Water Stewardship (AWS) Standard in order to promote a sustainable and feasible water use as the second certified AWS site in Italy. The implemented procedure, benefits and outputs of the AWS have transformed and are continuing to transform MTB's business and commitment towards a best-practice water management, with the aim to raise awareness and encourage others to do the same whilst conserving water as water stewards.
\end{abstract}

Keywords: Water Use, Sustainability, Overexploitation, Stewardship

\section{Introduction: A World-wide Water Crisis}

Water is a key resource and most essential element of life, yet less than 1\% of Earth's fresh water is usable. In 2019 the World Economic Forum (WEF) listed water scarcity, the lack of sufficient available water for usage demands, as one of the largest global risks of the $21 \mathrm{st}$ century [1]. Overuse, increasing demands, pollution, unsustainable management and changes in weather patterns due to global warming are key stressors that are affecting water availability and triggering an ever-growing water scarcity.

The WEF has reported water crises among the top five global risks for each of the past nine years and the United Nations foresee that two-thirds of the world's population will be living in water-stressed environments by 2030. The potential impact on the environment and humanity could lead to a water crisis that would face over 6 billion people, in conflict over water for basic needs. The sustainable management and conservation of this fundamental resource is a shared responsibility and a rapidly growing worldwide demand that faces every single country [2].

Italy, as most Mediterranean countries, is dominated by a temperate climate but with an uneven distribution of rainfall between north and southern regions, which does not allow a uniform water use or reserve. According to the Water Risk Filter [3], a tool designed by WWF to explore, assess, respond and value water risk around the globe, the Italian regions of Sicily, Puglia, Molise, Campagna, Toscana, and Emilia-Romagna are amongst those most subjected to high water depletion risk.

The Region of Emilia-Romagna is currently implementing numerous strategies in order to face water scarcity and promote a more sustainable use in the territory [4]. These strategies aim to promote and implement a more efficient and 
conscious use of water throughout the local population but also amongst water-demanding companies and activities [5]. The strategies can be summarized as follows:

(1) Technical actions based on changes in operational procedures or plant settings but also in household practices. At homes these practices may include the installation of toilet dams, faucet aerators and low-flow showerheads as well as the implementation of water-saving electrical appliances. In agriculture or in green urban area maintenance, the implementation of controlled, dip and micro-irrigation, as well as water efficient gardening and agricultural practices can be implemented in order to reduce water use. In industrial activities, the installation of water-saving technologies or plant settings as well as regular maintenance operations to verify leakages or abnormal water uses are amongst the best practices implemented;

(2) Behavioural actions based on changes of attitude towards everyday water saving strategies. These include the implementation of practices such as taking short showers instead of baths, running full loads of laundry, repairing leaky faucets indoors and out, buying water saving electrical appliance models and filling your sink or basin when washing and rinsing dishes;

(3) Reuse and recycling actions based on the implementation of reuse technologies and wastewater recycling systems. By identifying recycling opportunities and reuse modalities, a water demanding industry may considerably reduce its water use.

Many companies in Italy and around the globe have, in the last years, adopted a worldwide acclaimed certification procedure, the Alliance for Water Stewardship, for the implementation of best-practice strategies in order conserve and optimize the water resource and guarantee a future use to all.

Any responsible business should aspire to achieve a positive impact on the environment by using the water resource as sustainably as possible. The key is to look beyond a site's boundaries and consider its interaction in the catchment territory, as well as the related impacts to stakehold [6].

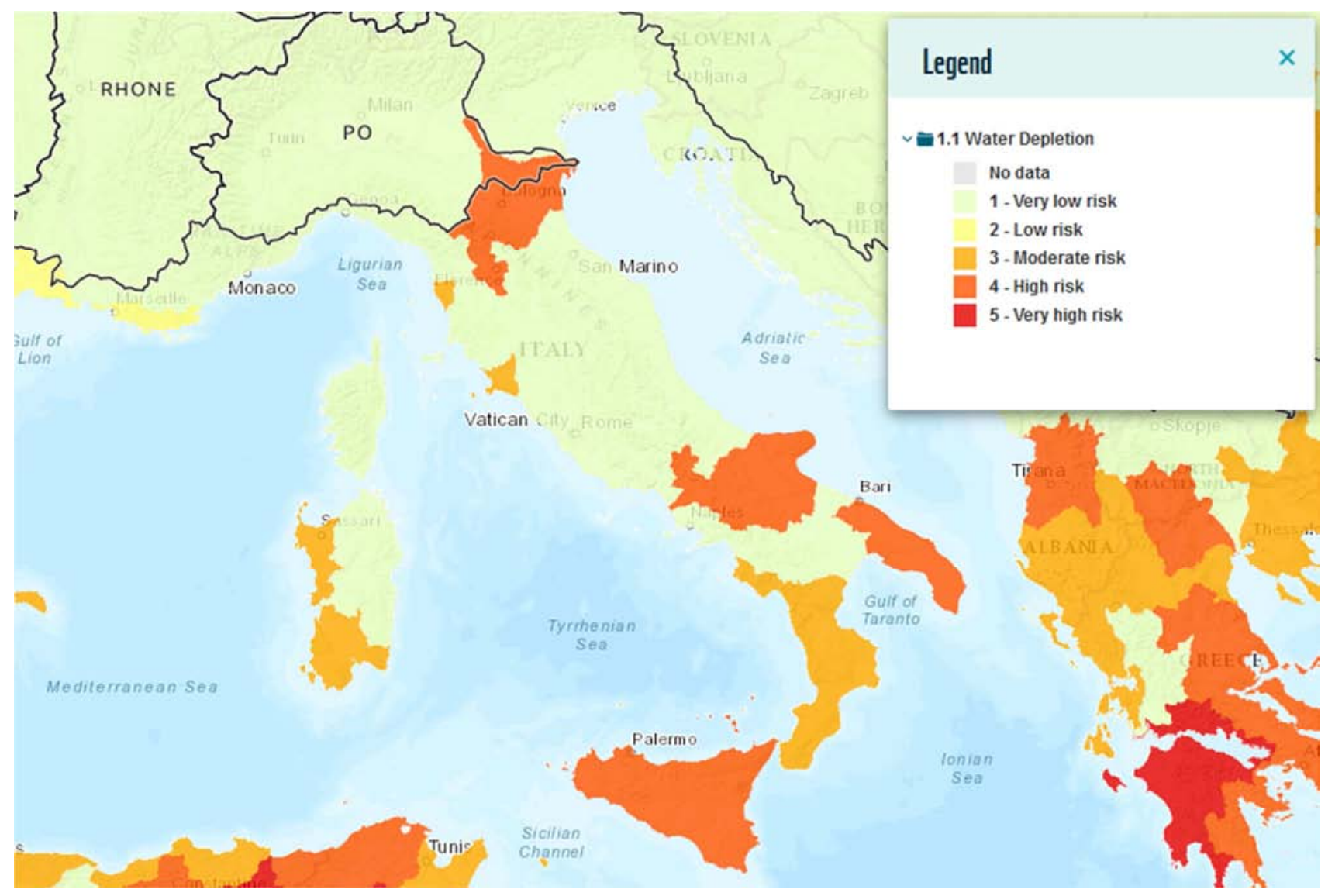

Figure 1. Water depletion in Italy according to the Water Risk Filter.

\section{The Alliance for Water Stewardship}

The Alliance for Water Stewardship (AWS) is the first global standard that assesses water-use across a 5-step certification procedure [7].

The AWS standard aims at promoting good water stewardship by assessing and consequently mitigating the physical, reputational and regulatory water-related risks associated to a certain site and its territory.

The implementation of the standard considers a variety of social, economic and environmental criteria, with the scope of implementing a sustainable water governance both on and off 
site as well as spreading good water use practices throughout the entire catchment area [8].

The 5 AWS steps may be summarized as follows:

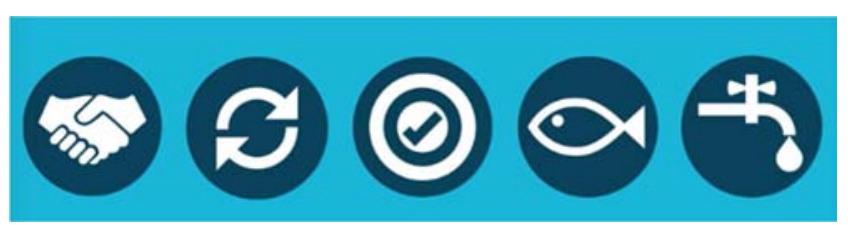

Figure 2. The AWS 5-step proceedure.

(1) Gather and Understand: gather data to understand shared water challenges and water risks, impacts and opportunities both on-site and in the catchment context, as well as the site's contributions (both positive and negative) to these factors;

(2) Commit and Plan: commit to be a responsible water steward and develop a water stewardship plan, in order to focus on how the site will act on shared water challenges, improve its performance and the status of its catchment in terms of AWS water stewardship outcomes;

(3) Implement: implement the plan outlined in Step 2 and ensure that the site is mitigating risks and driving actual improvements in performance;

(4) Evaluate: evaluate the site's performance against the actions taken in Step 3: learn from the results, both intended and unintended, and annually update that site's water stewardship plan based on the achieved outcomes;

(5) Communicate and Disclose: communicate about water stewardship and disclose the site's stewardship efforts: the disclosure of relevant information allows others to make informed opinions on the site's operations and tail their involvement to suit.

The implementation of the AWS standard will successively lead to the achievement of 5 outcomes, medium-termed results that reflect the motivations of why an organization sets about to implement the standard. The outcomes can be summarized as follows:

(1) Good Water Governance: the importance of water is recognized through laws, regulations, customs and management;

(2) Sustainable Water Balance: water is sufficient for human use, economic development and for the maintenance of healthy catchment ecosystems;

(3) Good Water Quality Status: water quality is good, enough to support environmental, economic, cultural and social needs;

(4) Important Water-Related Areas (IWRA): key water-related figures, such as wetlands or sites of cultural significance that play a key role in the nature and health of a catchment, are identified and conserved;

(5) Safe Water, Sanitation and Hygiene for all (WASH): safe access to water, sanitation and hygiene is provided as a fundamental human right and priority aspect for good water stewardship.

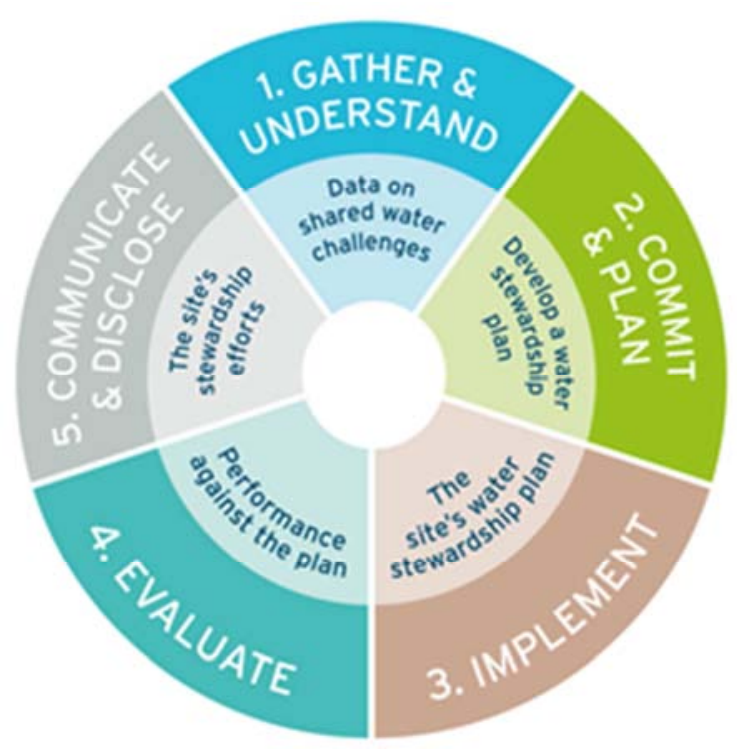

Figure 3. The AWS outcomes.

The AWS standard offers a credible, globally applicable framework for water users to understand their own water use and impacts, and to work collaboratively and transparently with others for sustainable water management within their territory [5]. With the aim to sustainably manage the water resource, AWS is proving to be an innovative certification adopted by a wide range of multinational companies in order to reduce their water footprint. Coca-Cola, Nestlè and Philip Morris International, are amongst those that have implemented AWS with the scope of certifying their production sites and transforming their business by working together towards a sustainable future [9].

Philip Morris International (PMI) has always adopted ambitious sustainability initiatives as core of their business strategy, and firmly believes that working efficiently goes hand in hand with helping and managing the environment. Since 2017 PMI's environmental milestones include an active commitment to responsible and sustainable water management by piloting the AWS standard. After the success of the pilot factory, the AWS certification was launched to all PMI affiliates world-wide, with a target of certifying all main operations by 2025. PMI is assisted and aided in the certification procedure by the environmental consultants Denkstatt and HPC of the INOGEN International Alliance.

Philip Morris Manufacturing \& Tecnology Bologna (MTB), located in the Italian region of Emilia-Romagna, is a global lead site for the large-scale production of HeatSticks, reduced risk products (RRP) used in the IQOS tobacco heating system. MTB is certified AWS since July 2019, is the first ever RRP site in the world to be certified AWS and the secondo certified site in Italy after the Sicilian affiliate of Nestlé. 


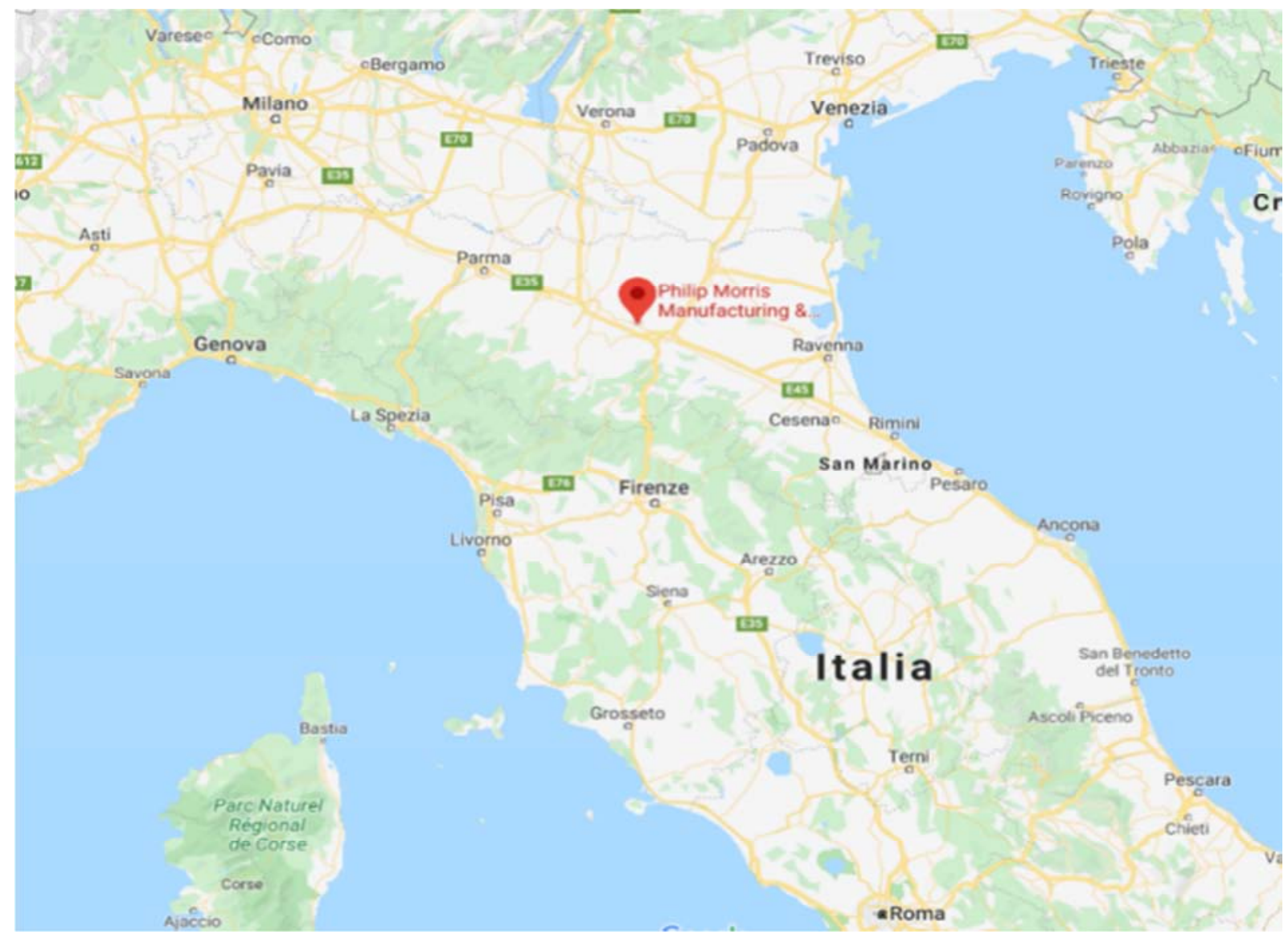

Figure 4. Location of the Philip Morris Manufacturing \& Tecnology Bologna affiliate in Italy.

MTB is transforming their business and their commitment towards a best-practice water management. The AWS standard abled MTB to not only follow the AWS 5 step proceedure towards a Core-Level certification but also become a water steward, that is a sustainable water-user with the scope of reducing potable water consumption and impact on the territory [10].

The AWS Standard allowed MTB to understand, identify and implement several AWS principles:

(1) Identification of the catchment area: the catchment area is the land that the site directly affects and is reliant upon. MTB's catchment area was defined together with its potable water supplier, which draws water from rivers and deep aquifer bodies;

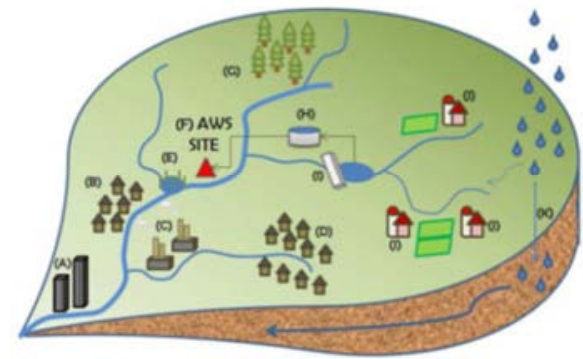

Figure 5. Catchment area delimitation.

(2) Understanding and prioritizing water-related risks: MTB is in the Italian region of Emilia-Romagna and consequently subjected to a high baseline water stress due to the presence of many water-demanding industries, high competition between users and an estimated decrease in precipitation;

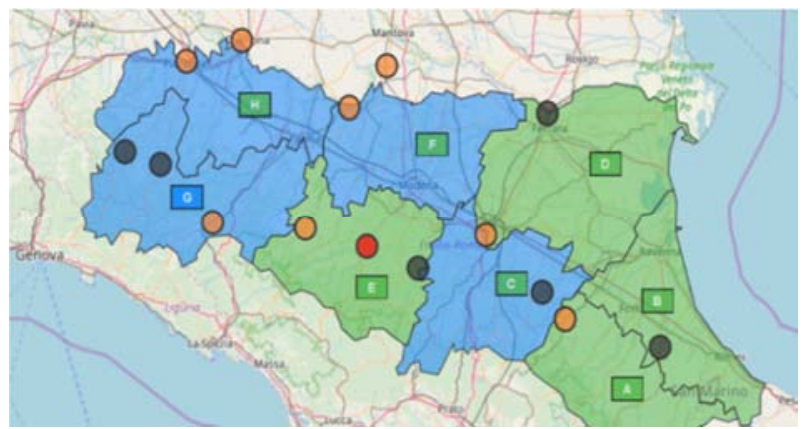

Figure 6. Below average cumulated precipitations (in orange) in the Bologna area.

(3) Identification of IWRA, sensitive areas of high conservational value: MTB identified IWRA in its catchment area and defined their status in order to evaluate their health and dynamics of the water resource;

(4) Active outreach to stakeholders and participation in catchment governance: MTB created mutual collaborations with stakeholders such as the local community, environmental authorities, service providers, water demanding companies, and 
environmental federations. MTB also implemented multiple awareness activities in order to increased public awareness on catchment water-related risks and promote water-saving efforts;

(5) Maintain and improve direct and indirect water use within the catchment: MTB implemented initiatives and strategies in order to reduce potable water consumption on-site and consequently remove less water from the catchment area and sensitive environments. By recycling wastewater in plant processes, implementing water-saving settings and introducing new and innovative water-saving technologies, in 2019 MTB obtained a $20 \%$ water saving per million of HeatSticks produced;

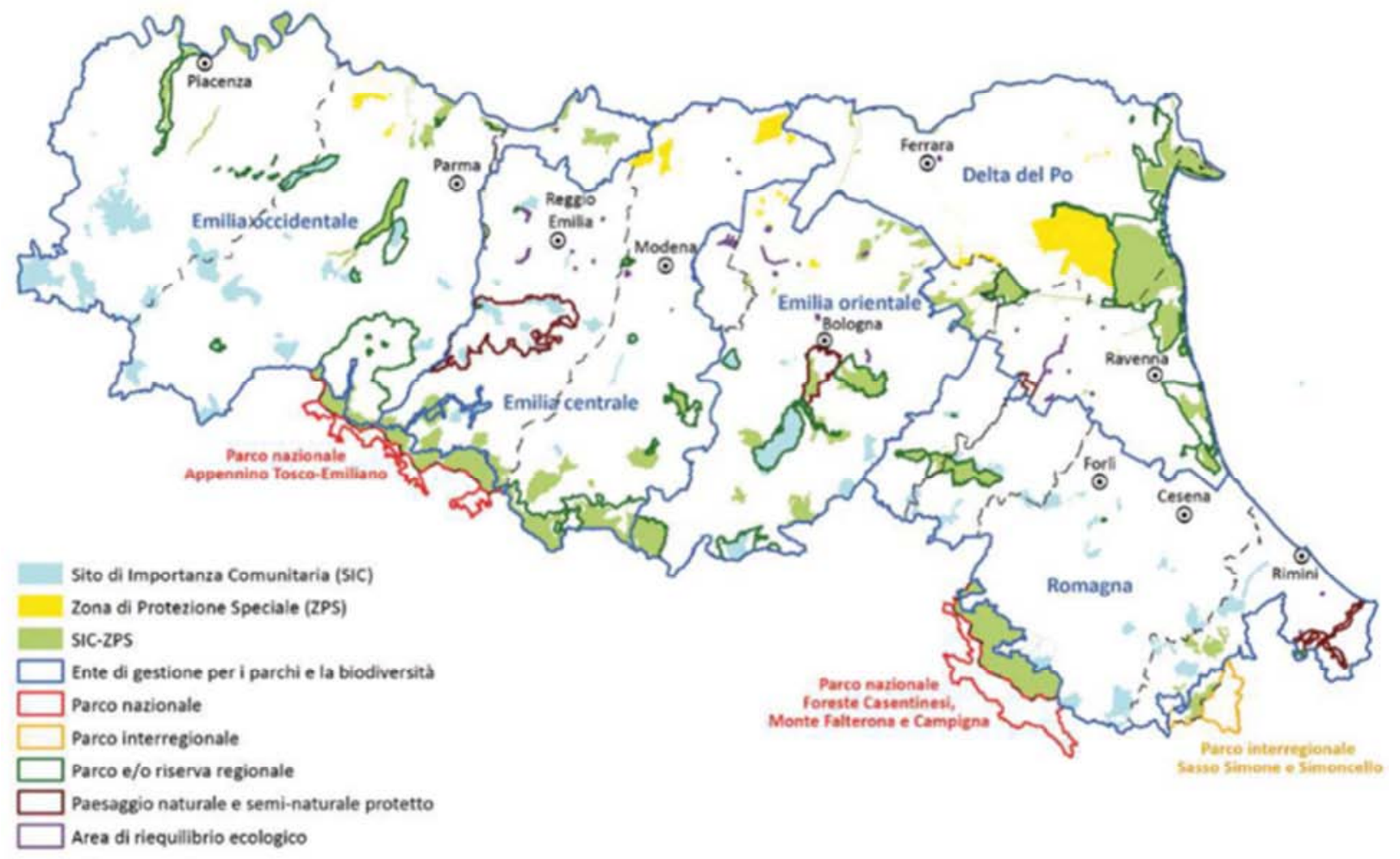

Figure 7. Identification of IWRA in the Emilia-Romagna Region.

(6) Increase awareness of water issues and disclosure of best-practice performance: MTB strongly believes in effective communication in order to raise awareness for a specific topic or situation. The AWS philosophy has been disclosed to every employee through water sensibilization presentations, internal communications and newsletters [11].

MTB has actively raised public awareness on water sustainability practices and committed towards catchment wellbeing by engaging in events and campaigns with stakeholders and communities. The implementation of the AWS standard by MTB has been disclosed in various ways, for example social medias, public events, press campaigns, congresses and technical tables. Increased public awareness on water issues as well as sharing goals and best practices is fundamental to improve local collaboration but also disclose the AWS philosophy and values. MTB aims at setting a positive example on how other companies could implement a more conscious and sustainable business strategy.

\section{Transforming our Business}

The AWS standard certifies a site's commitment to responsible water management and sustainable innovation for the wellbeing of the environment and future generations. Specifically, the AWS journey abled MTB to minimize its water footprint by understanding its impact and the risks associated to water use in the catchment context. By working collaboratively with its employees and other local stakeholders, the AWS delivered positive impacts both internally and externally [12].

The implementation of the AWS Standard enabled MTB to contribute to good water stewardship practices that not only optimized and reduced water use on-site, but also contributed to wider sustainability goals throughout the entire territory. The path is still ongoing, but MTB and PMI are proudly transforming their business towards a sustainable future with the hope that their commitment towards the environment with be followed by other water stewards around the world.

The benefits that any business reality may receive from implementing the AWS Standard are numerous and include site, catchment and organizational-level benefits. These may be summarized as follows:

(1) Increased water-use efficiencies;

(2) Increased water-related risk mitigation;

(3) Growth in staff capacity and motivation; 
(4) Strengthened stakeholder relationships;

(5) Building and growing community trust;

(6) Improvement of local water quality;

(7) Protection of IWRAs;

(8) Enhanced local collaboration;

(9) Supported knowledge sharing;

(10)Strengthened multi-stakeholder governance;

(11)Understanding of site's risks;

(12)Common approach in water governance;

(13)Credible independent claims;

(14)Support for CSR;

(15)Contribution to CDP water at Global Level;

(16)Reinforcement of sustainability credentials;
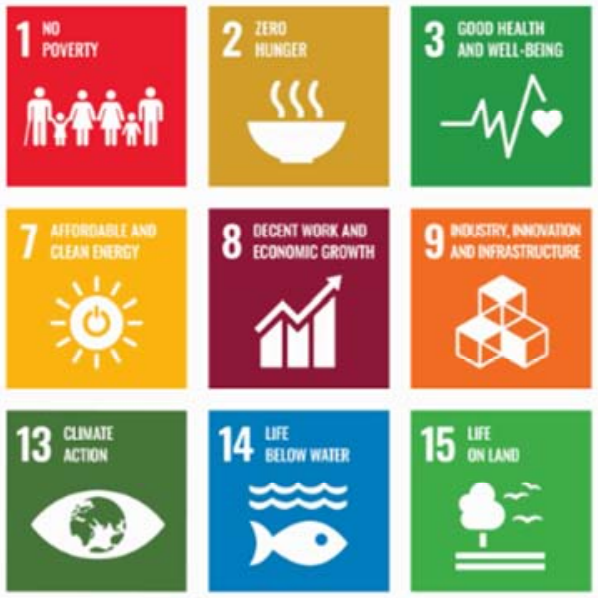
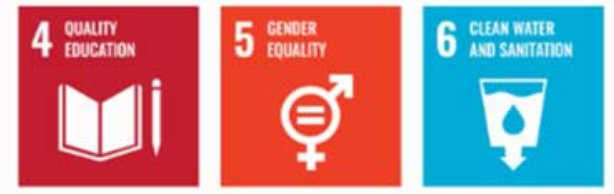
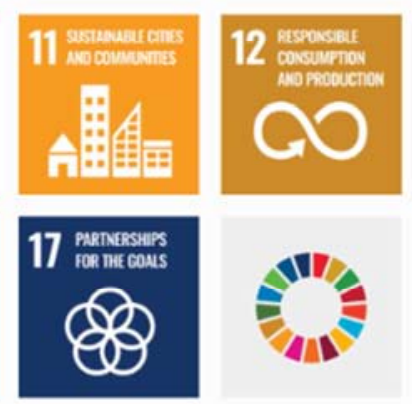

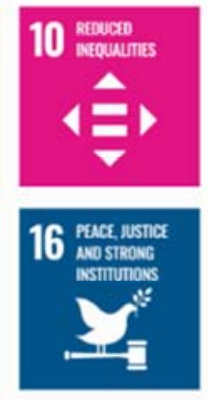

(17)Higher ranking in CDP water at Global Level;

(18)Reinforcement of brand's sustainability credentials.

The continuous work in progress which characterize which it is located.

As ultimate goal, the AWS certification aims to deliver a positive impact to support a water-secure world that enables prosperity for people cultures, business and nature. In particular, its implementation meets one of the 17 Sustainable Development Goals developed by the United Nations in 2015, that it goal number 6 Clean Water and Sanitation.

Figure 8. Sustainable development goals of the UN.

\section{Conclusion}

The AWS Standard implemented in the MTB facility offered a credible, globally applicable framework to understand water use and impacts, address challenges and risks as well as engage collaboratively and transparently with other local stakeholders towards a sustainable water management within a wider catchment context.

The AWS certification procedure offers, through its entire implementation, the possibility to understand how water behaves and its associated risks as well as collaborating and sharing knowledge in a global, multi-stakeholder consultation process that is open, transparent and consensus based.

In the future MTB and at global level PMI, will continue to implement sustainable water practices and raise awareness in order to encourage others to do the same. Water is a precious and shared resource that must be preserved and conserved for us and for all future generation at a global dimension.

\section{References}

[1] The United Nations, "Water: a shared responsibility" World Water Development Report 2, 2006.
[2] Wang X. C., Zhang C., Ma X., Luo L., "Water Cycle Management", 2015.

[3] Weblink:

https://waterriskfilter.panda.org/en/Explore/CountryProfiles\#o verview/150.

[4] A. Guerrini, G. Romano, "Water Management in Italy", 2014.

[5] D. H. Chen, "Sustainable Water Management", 2016.

[6] J. Holden, "Water Resources: An Integrated Approach", 2013.

[7] Weblink: https://a4ws.org/.

[8] W. Sarni, D. Grant "Water Stewardship and Business Value", 2018.

[9] W. Sarni, "Water Stewardship and Business Value: Creating Abundance from Scarcity”, Routledge, 2018.

[10] P. Gleick, H. Cooley et al., "The Worlds Water, Volume 9: The Report on Freshwater Resources", Pacific Institute for Studies in Development, Environment, and Security, Oakland, California, 2018.

[11] W. Whipple Jr, "Water Resources: A new Era for Coordination", ASCE Press, 1998.

[12] M. Seneviratne, "A Practical Approach to Water Conservation for Commercial and Industrial Facilities", Elsevier Science, 2006. 